\section{Archived Guthrie blood spots as a novel source for quantitative DNA methylation analysis}

\author{
Nicholas C. Wong, Ruth Morley, Richard Saffery, and Jeffrey M. Craig \\ Developmental Epigenetics, Murdoch Childrens Research Institute, \\ Royal Children's Hospital, Department of Paediatrics, University of Melbourne, \\ Parkville, Victoria, Australia
}

BioTechniques 45:423-430 (October 2008)

doi $10.2144 / 000112945$

\begin{abstract}
Sodium bisulfite treatment followed by PCR and DNA sequencing is widely considered the gold standard for the analysis of DNA methylation patterns. However, this technique generally requires substantial quantities of genomic DNA as starting material and is often associated with degradation of DNA. Here, we assess the feasibility of performing bisulfite sequencing on DNA isolated from 3-mm diameter punches of dried blood Guthrie spots. We demonstrate that it is possible to perform bisulfite sequencing from both freshly prepared and archived dried blood spots, using a combination of high purity DNA extraction and efficient bisulfite conversion. With the number of new technologies available for DNA methylation studies, we have extended this analysis and have successfully used a highthroughput mass spectrometry method for DNA methylation analysis on these samples. This provides a new source of material for epigenetic analysis of birth samples and provides an invaluable reference point to track temporal change in epigenetic profiles possibly linked with health and disease.
\end{abstract}

\section{INTRODUCTION}

Dried blood or "Guthrie" spots are taken from children born in developed countries for newborn screening for a number of metabolic disorders including phenylketonuria (PKU) (1) and genetic testing using PCR-based assays (2). However, the utility of such samples for DNA methylation analysis has remained unclear due to the general requirement of microgram quantities of genomic DNA as starting material (3). More recently, bisulfite sequencing on limited amounts of DNA from microdissected samples has been demonstrated (4). Bisulfite sequencing is capable of discriminating methylated cytosine from unmethylated cytosine in genomic DNA (3). However, traditional bisulfite-based DNA modification has the inherent problem of DNA degradation due to the destructive nature of the modification process (5). Often, this has deleterious effects on the ability to successfully generate test PCR products for analysis. Furthermore, there is evidence for DNA fragmentation over time with storage of dried blood spots (6), and this can also influence the efficiency of any downstream, PCR-based applications. More recently, matrixassisted laser desorption ionization time-of-flight (MALDI-TOF) mass spectrometry has been used as a means of high-throughput DNA methylation analysis (7) and could potentially be used to analyze such samples en masse. The degree to which these factors will influence the utility of bisulfite-based methylation analysis on Guthrie spots has not previously been tested.

Here, we aim to investigate the utility of DNA methylation analysis by bisulfite PCR-based DNA sequencing and SEQUENOM analysis on dried blood spot Guthrie samples. We gene promoters, predicted to be either methylated [DNA methyltransferase 3-like (DNMT3L) gene promoter] or unmethylated [vitamin D receptor (VDR) gene promoter], by bisulfite PCR and sequencing of freshly prepared and aged, dried blood Guthrie spots. We further demonstrated the utility of this approach by examining the methylation levels at the H19 imprinted region using the SEQUENOM MassArray analyzed the methylation status of two
EpiTYPER analysis. The demonstration of archived DNA blood spots as a suitable template for DNA methylation analysis represents a unique resource for the retrospective analysis of epigenetic status of gene regulatory region methylation in whole blood specimens at birth.

\section{MATERIALS AND METHODS}

Seven genomic DNA extraction kits using a range of extraction methods including resin-based [Instagene Matrix and Chelex 100 (both from Bio-Rad Laboratories, Hercules, CA, USA)], lysis-based [Instagene $\mathrm{D}_{\mathrm{x}}$ (Bio-Rad Laboratories), ExtractN-Amp (Sigma-Aldrich, St. Louis, MO, USA), Blood DNA Mini kit (Qiagen, Hilden, Germany), and ReadyAmp DNA Purification System (Promega, Madison, WI, USA)], or magnetic beadbased (ChargeSwitch Forensic DNA Purification kit; Invitrogen, Carlsbad, CA, USA) were used on 3-mm punches of freshly prepared dried blood Guthrie spots (Table 1). Where indicated, modification of the recommended protocol for dried blood spots was also carried out according to manufacturer's instructions.

Sequence tagged site (STS) marker PCR amplification of D6S510 and D6S265 (primers listed in Table 2) was performed on $1 \mu \mathrm{L}$ extracted genomic DNA. PCR Mastermix (Promega) was used to amplify the STS markers, and the conditions used for amplification are as follows: denaturation at $95^{\circ} \mathrm{C}$ for 2 min, followed by 25 cycles of $95^{\circ} \mathrm{C}$ for $30 \mathrm{~s}, 53^{\circ} \mathrm{C}$ for $30 \mathrm{~s}$, and $72^{\circ} \mathrm{C}$ for $30 \mathrm{~s}$.

The yield of genomic DNA from each extraction method was determined by spectrophotometry (Nanodrop, Wilmington, DE, USA) and was further characterized by using a serial 2 -fold standard curve and absolute quantitative PCR using primers to D6S510 and SYBR GreenER qPCR Supermix (Invitrogen, Carlsbad, CA, USA) using the following amplification conditions: polymerase activation at $95^{\circ} \mathrm{C}$ for $30 \mathrm{~s}$, $53^{\circ} \mathrm{C}$ for $10 \mathrm{~min}$, followed by 40 cycles of $95^{\circ} \mathrm{C}$ for $15 \mathrm{~s}, 60^{\circ} \mathrm{C}$ for $60 \mathrm{~s}$. Quantitative PCR was analyzed using an ABI 7300 (Applied Biosystems, Foster City, CA, USA). 
Four bisulfite modification methods were then evaluated on the extracted DNA in addition to a commonly used in-house method (based on Reference 3): EZ DNA Methylation kit (Zymo Research, Orange, CA, USA), CpGenome DNA Modification kit (Millipore, Billerica, MA, USA), and MethylEasy kit (Human Genetic Signatures, Sydney, Australia). Protocols were performed according to the manufacturer's instructions for the minimum amount of genomic DNA as starting material recommended for each kit.

Traditional bisulfite PCR was performed using a two-round hemi-
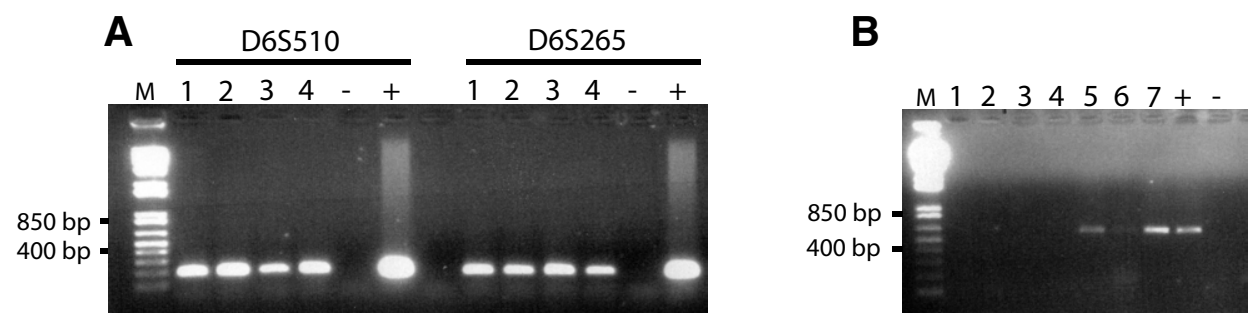

Figure 1. Microsatellite and bisulfite PCR analysis. (A) Successful PCR amplification of two STS markers D6S510 and D6S265 from genomic DNA isolated from 3-mm punch dried blood Guthrie spots using four out of the seven DNA extraction kits. Lane 1, Instagene Matrix; lane 2, Chelex 100 Resin; lane 3, ReadyAmp Genomic DNA Purification System; lane 4, ChargeSwitch Forensic DNA Purification kit; -, negative control; +, positive control. (B) Bisulfite PCR amplification of the vitamin $D$ receptor promoter of DNA samples extracted by different methods after MethylEasy kit conversion. Lane 1, Extract-N-Amp; lane 2, Blood DNA Mini kit; lane 3, Instagene Matrix $D_{x}$; lane 4, Chelex 100 Resin; lane 5, Instagene Matrix; lane 6, ReadyAmp Genomic DNA Purification System; lane 7, ChargeSwitch Forensic DNA Purification kit; +, positive control; -, negative control. nested strategy. Bisulfite primers were targeted to the VDR and DNMT3L gene promoter (Table 2). PCR Mastermix (Promega) was used for amplification, and the conditions were $95^{\circ} \mathrm{C}$ for 2 min, followed by 30 cycles of $95^{\circ} \mathrm{C}$ for $30 \mathrm{~s}, 52^{\circ} \mathrm{C}$ for $30 \mathrm{~s}, 72^{\circ} \mathrm{C}$ for $90 \mathrm{~s}$, and a final extension at $72^{\circ} \mathrm{C}$ for $7 \mathrm{~min}$. The first round of PCR was performed using the Bi_VDR1F and Bi_VDR1R primers with $1 \mu \mathrm{L}$ amplification reaction transferred to a second round of PCR using the Bi_VDR1F and Bi_VDR2R primers. Similarly, Bi_DNMT3L1F and Bi_DNMT3L1R were used for the first round of PCR, and Bi_DNMT3L2F and Bi_DNMT3L1R were used for the second round of PCR.

The products were subjected to cloning and DNA sequencing by ligation into pGEMT-Easy vector (Promega) with transformation into DH5- $\alpha$ Escherichia coli cells. Positive clones were selected for automated fluorescent sequencing (see Figure 2, A and B)

Table 1. Evaluation of Guthrie Spot DNA Extraction Methods and Bisulfite Conversion Methods for Bisulfite PCR and Sequencing

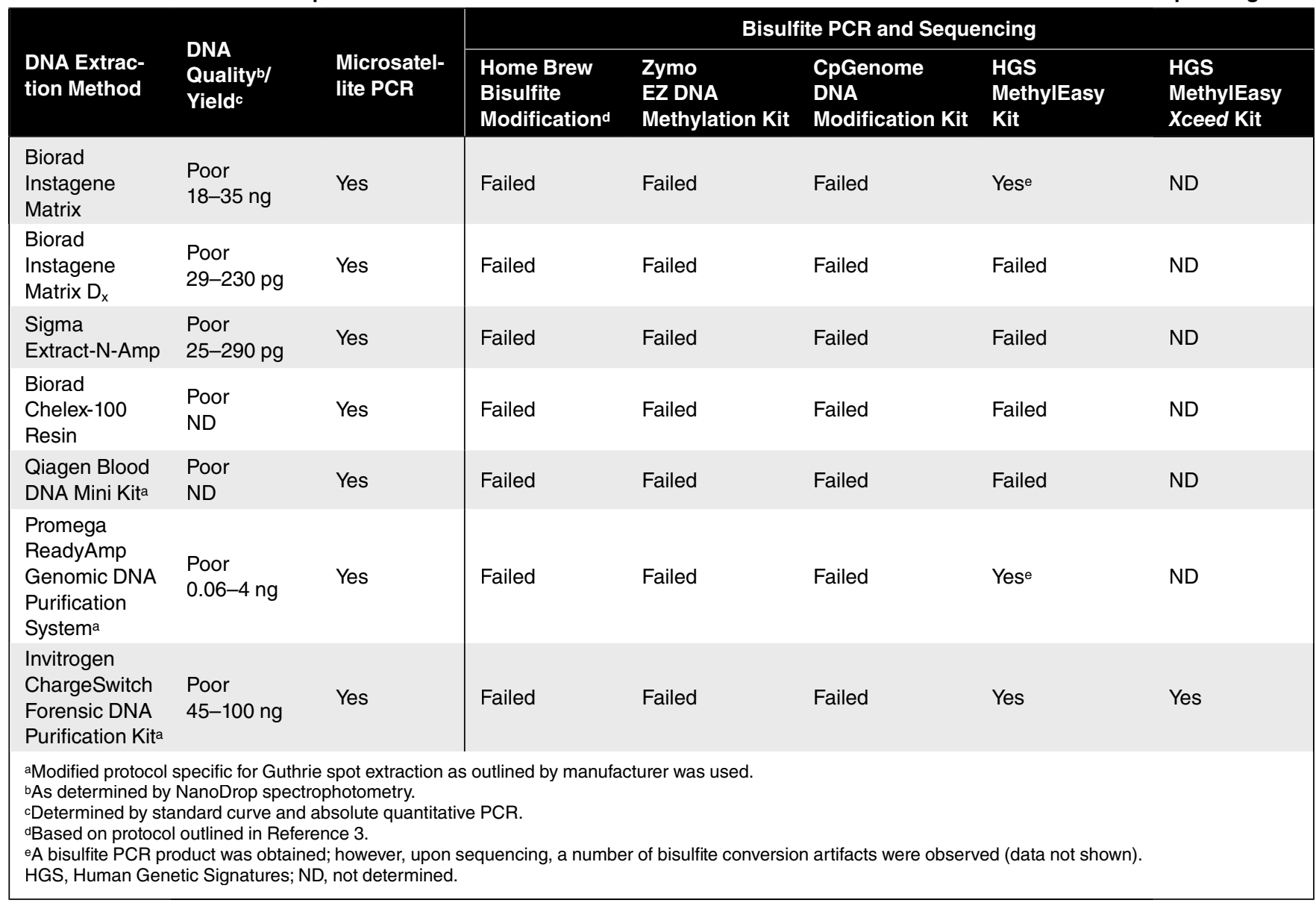


by the Australian Genome Research Facility (www.agrf.org.au). Methylation of individual $\mathrm{CpG}$ sites was determined using BiQ Analyzer (8).

For the SEQUENOM mass spectrometry method of analysis, tagged bisulfite primers (Sqnm_163bp1F and Sqnm_163bp1R) (Table 2) to the H19 imprinted locus (9) were used in modified bisulfite PCR with PCR Mastermix (Promega), and the following amplification conditions were used: initial denaturation at $95^{\circ} \mathrm{C}$ for $2 \mathrm{~min}$, followed by 5 cycles of $95^{\circ} \mathrm{C}$ for $30 \mathrm{~s}, 56^{\circ} \mathrm{C}$ for $30 \mathrm{~s}$, $72^{\circ} \mathrm{C}$ for $90 \mathrm{~s}$, then 35 cycles of $95^{\circ} \mathrm{C}$ for $30 \mathrm{~s}, 60^{\circ} \mathrm{C}$ for $30 \mathrm{~s}, 72^{\circ} \mathrm{C}$ for $90 \mathrm{~s}$, and a final extension at $72^{\circ} \mathrm{C}$ for $7 \mathrm{~min}$.

A methylation titration was constructed with $0 \%, 25 \%, 50 \%, 75 \%$, and $100 \%$ DNA methylation ratios using genomic DNA isolated from a leukemia cell line, REH (Accession no. CRL8286; ATCC, Manassas, VA, USA), which we have found to be unmethylated at the H19 differentially methylated region (DMR) and in vitro-methylated human genomic DNA (Millipore). Each titration (100 ng) was bisulfite-converted using the MethylEasy Xceed Bisulfite Modification kit (Human Genetic Signatures) according to manufacturer's instructions and subjected to PCR amplification of the H19 imprinted locus (Sqnm_163bp1F and Sqnm_163bp1R) (Table 2) for SEQUENOM mass spectrometry analysis.

Products derived for SEQUENOM mass spectrometry were subjected to the homologous MassCLEAVE reaction chemistry (hMC; SEQUENOM, San

A

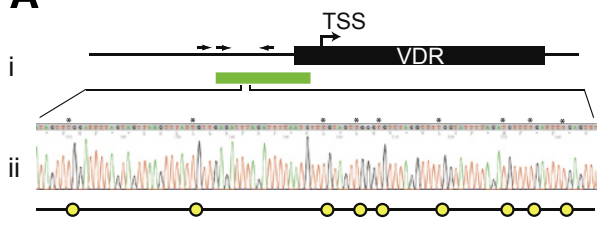

B
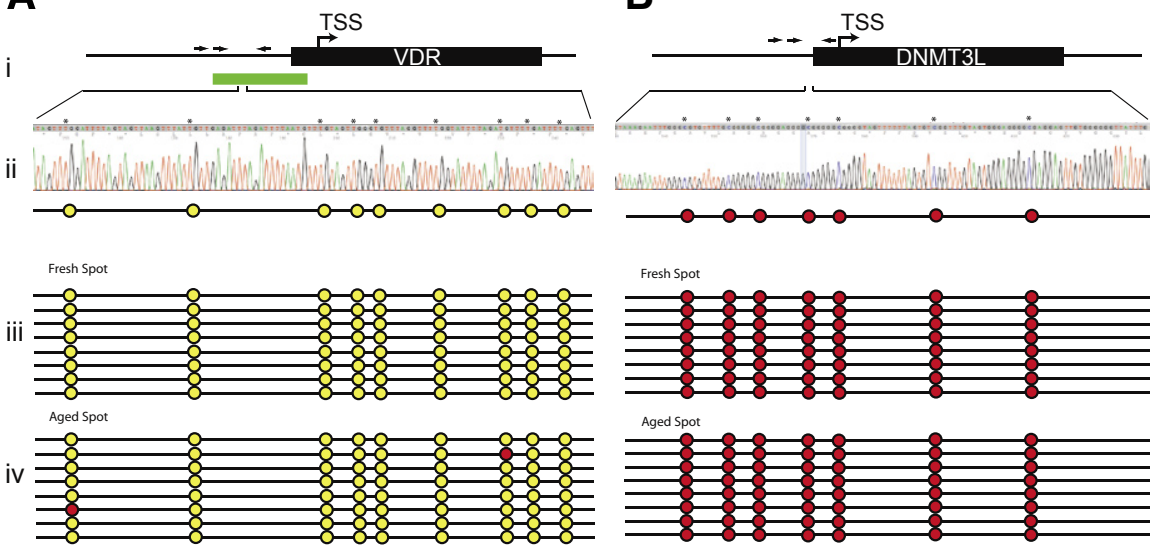

Figure 2. Bisulfite PCR and sequencing analysis. (A) (i) Depiction of promoter structure of vitamin D receptor (VDR). Location of bisulfite PCR primers (arrows), transcription start site (TSS), and CpG island (green) are depicted. (ii) Representative DNA sequence chromatogram of the VDR promoter from clones generated by bisulfite PCR in this study (chromatogram viewer used was 4Peaks; mekentosj. com). Methylation was scored for each allele at the individual CpG dinucleotide sites (*) as methylated (red) or unmethylated (yellow). Eight clones derived from (iii) freshly prepared dried Guthrie spots and (iv) eight clones from aged Guthrie spots (up to 5 years) were sequenced. (B) (i) Cartoon depiction of promoter structure of DNA methyltransferase 3-like (DNMT3L) gene. Location of bisulfite PCR primers (arrows) and the TSS are depicted. DNMT3L promoter does not contain a CpG island. (iiiv) Representative bisulfite PCR and sequencing results from the DNMT3L promoter depicting hypermethylation of this promoter from both fresh and aged, dried blood Guthrie spots.

Diego, CA, USA) according to manufacturer's instructions. The T-cleavage reaction was performed on the samples that were then spotted using the SEQUENOM Nanodispenser onto a SpectroCHIP for subsequent analysis.

\section{RESULTS AND DISCUSSION}

Here, we have investigated the possibility of performing bisulfite-mediated DNA methylation analysis by either bisulfite PCR and sequencing or mass spectrometry on DNA derived from dried blood spots. We tested a range of commercially available DNA extraction kits for purifying genomic DNA from fresh and dried blood for downstream PCR applications and applied this to DNA methylation analysis. Furthermore, we have evaluated a number of commercially available bisulfite-based conversion kits alongside the in-house bisulfite conversion method (3) on their utility for DNA methylation analysis on dried blood Guthrie samples.

By spectrophotometry, all methods of genomic DNA extraction revealed generally very low quality (absorption

Table 2. Primers Used in This Study

\section{Primer}

D6S510F

D6S510R

D6S265F

D6S265R

Bi_VDR1F

Bi_VDR2F

Bi_VDR1R

Bi_DNMT3L1F

Bi_DNMT3L2F

Bi_DNMT3L1R

Sqnm_163bp1F

Sqnm_163bp1R

\section{Sequence}

5'-AATGGGCTACTACTTCACACC-3'

5'-CAACACACTGATTTCCATAGC-3'

5'-AGTCACCCTACTGTGCTATC-3'

5'-ATCGAGGTAAACAGCAGAAAG-3'

5'-AATATTTTTTTGTTGTTTAAGTGTTAAGTAT-3'

5'-TAAATTTTAGTGTTTTTTAGTGTTTTAGTT-3'

5'-CTAACCTAATCAACCCAAATAAAAATA-3'

5'-GTTTTGGTTAGTCGTATTTTTGAAGGTAGT-3'

5'-AGTAGGAAAGAGATTTATAGGGTTGTTGTT-3'

5'-AACTCTTAACCCTCCCCACCTAAAAA-3'

5'-AGGAAGAGAGTGAGGTGAATTTTAGGGATTGTAGG-3'

5'-CAGTAATACGACTCACTATAGGGAGAAGGCTACCTCACAAACTCTCCCAAACC-3'

\section{$\mathbf{T}_{\mathrm{m}}\left({ }^{\circ} \mathbf{C}\right)$}

55.9

55.8

55.7

56.3

54.8

53.2

54.8

56.0

56.0

56.0

$56.0 / 60.0$ a

$56.0 / 60.0^{a}$

aPrimer sequences from Reference 9 .

$\mathrm{T}_{\mathrm{m}}$, melting temperature. 


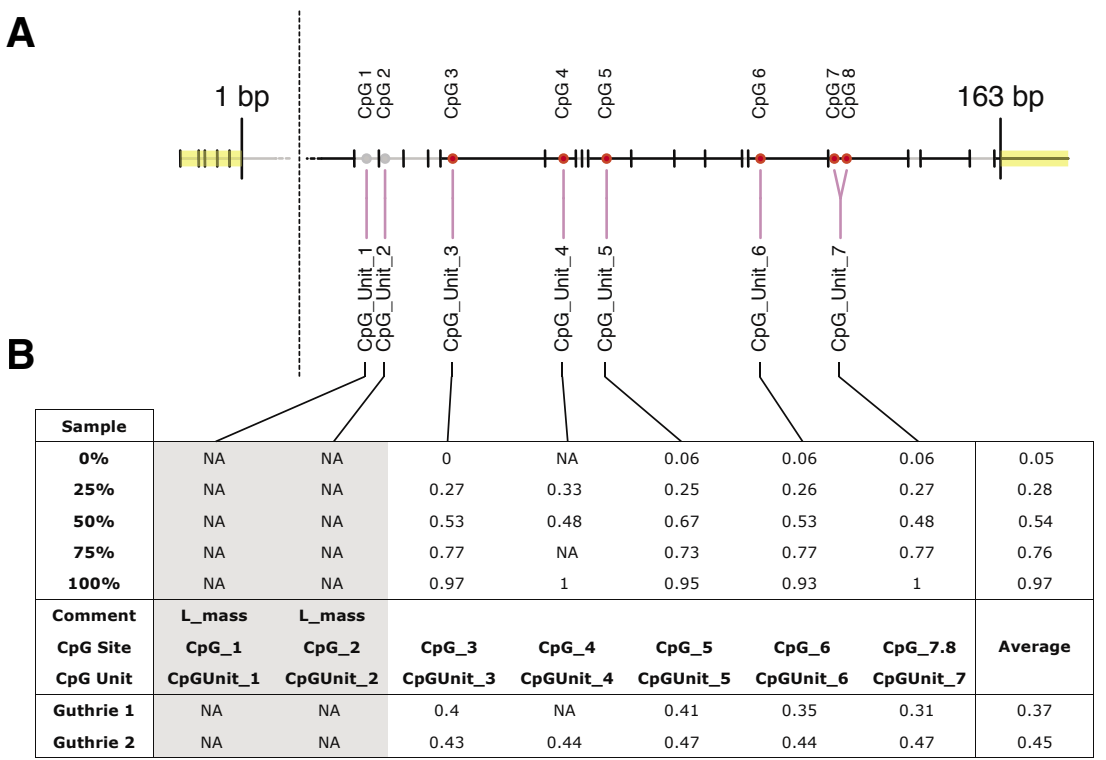

Figure 3. SEQUENOM MassArray EpiTYPER analysis. (A) Depiction of H19 assay (9) used in this study. Diagram depicts the CpG sites analyzed within the assay by SEQEUNOM MassArray EpiTYPER (red circles), while sites that are not analyzed are depicted as gray circles. The vertical bars along the product depict the sites of T-fragmentation caused by the MassCLEAVE reaction. Diagram adapted from an R-Script program in (17). (B) Table displaying the methylated ratios at each CpG unit by SEQUENOM EpiTYPER software. NA, not analyzed.

260/280 ratios ranging from $0-0.5$ ) and low yield of genomic DNA from the dried blood spots, which may be due to carryover contaminants from the blood (6) (Table 1). Genomic DNA was detected from all extraction methods by standard D6S510 and D6S265 microsatellite PCR (Figure 1A). By quantitative PCR, all extraction methods yielded between $0.025-100$ ng of total genomic DNA (Table 1). Our comparisons reveal that genomic DNA extracted with the ChargeSwitch Forensic DNA Purification kit yielded the highest amounts of genomic DNA (Table 1).

The genomic DNA extracted from these kits was then subjected to bisulfite modification, PCR, and sequencing. Products were successfully obtained on bisulfite-converted DNA generated using the MethylEasy kit with DNA extracted using Instagene Matrix, ReadyAmp DNA Purification System, and ChargeSwitch Forensic DNA Purification kits, while other bisulfite conversion methods failed to yield analyzable products (Table 1 and Figure 1B). Conversion artifacts (detection of non-CpG methylation after bisulfite PCR and sequencing) were observed in clones derived from DNA extracted by the Instagene Matrix and ReadyAmp kits (Table 1) making these methods of extraction inappropriate for subsequent DNA methylation analysis (data not shown). From this result, we have determined that genomic DNA extracted from dried blood spots using the ChargeSwitch Forensic DNA Purification kit and subsequently bisulfite-modified using the MethylEasy kit to be suitable for downstream DNA methylation analysis.

We then tested this method on dried blood Guthrie spots prepared up to 5 years ago, that were stored according to standard procedures for newborn screening laboratories, with similar success to freshly prepared samples (Figure 2, A and B). Furthermore, we tested a second-generation bisulfite modification kit (MethylEasy Xceed kit) that considerably reduced modification time from $16 \mathrm{~h}$ to $45 \mathrm{~min}$ with similar success (Table 1 ).

Using this approach, we observed that the VDR promoter was unmethylated (Figure 1C) in all dried blood spots tested and was consistent with ubiquitous VDR expression in normal tissue (8). In contrast, the DNMT3L promoter was observed to be methylated in the same samples consistent with previous data, suggesting a highly restricted expression profile for DNMT3L and gene regulation by a mechanism that includes promoter hypermethylation (10).

We then subjected the same aged samples to SEQEUNOM MassArray EpiTYPER analysis using primers targeting the H19/IGF2 imprinted region (Table 2 and Figure 3A) (9). A standard titration of DNA methylation was generated using premixed ratios of unmethylated DNA to in vitromethylated human genomic DNA. One hundred nanograms of these titrations were then subjected to the same bisulfite treatment and PCR before being analyzed by SEQUENOM EpiTYPER software. The expected fragmentation and analyzable $\mathrm{CpG}$ sites/units are shown in Figure 3A. Two $\mathrm{CpG}$ units (Figure 3A, units 1 and 2) were not analyzed, as these fragments fell outside the analysis mass window range. The remaining $\mathrm{CpG}$ units (units 3-7 and 8) were called by EpiTYPER for the titration samples, and the two aged Guthrie samples were analyzed. The methylation ratios analyzed for the titration were within the manufacturer's specifications (5\% coefficient of variation) and generally agree with the premixed ratios of DNA methylation. DNA methylation was also analyzed from the aged Guthrie spots described above and showed an average methylation ratio of analyzed $\mathrm{CpG}$ units across the entire product of 0.37 and 0.45 (Figure 3B). This observation is in keeping with recently published observations of the same locus using the same methods on more readily available and abundant specimens including blood (11).

In this study, we have demonstrated the utility of bisulfite-based DNA methylation analysis of DNA extracted from dried blood Guthrie spots. We have demonstrated that the combination of genomic DNA extraction using the ChargeSwitch Forensic DNA Purification kit with subsequent bisulfite modification using the MethylEasy kit yields DNA of sufficient quantity and quality for amplification and DNA sequencing of test genomic regions of interest. DNA extracted from all kits tested was of sufficient quality and yield for general genomic PCR ampli- 
fication as intended by the respective manufacturers. The ChargeSwitch kit was the only extraction method that used magnetic beads, which facilitates the removal of more contaminants and is a critical factor for efficient bisulfite modification (5). With the exception of the MethylEasy kit, the other three methods of bisulfite modification of DNA failed, possibly due to the nature of bisulfite conversion, where excessive degradation of the starting DNA is often observed (5). The MethylEasy kit uses a proprietary "lossless" method, in which the starting DNA is preserved throughout the modification process (www.geneticsignatures.com).

We have determined that the DNA isolated in this manner is of sufficient quality and quantity for downstream bisulfite PCR-mediated DNA methylation analyses, including DNA sequencing and SEQUENOM MassArray EpiTYPER analysis. We have shown that it is possible to detect unmethylated and methylated gene promoters by cloning and sequencing and that is possible to detect imprinted regions from such samples using SEQUENOM. From the titration results, SEQUENOM would be the method of choice to evaluate regions that are not completely methylated or not completely unmethylated, because it is not prone to potential bias that could arise from PCR cloning and sequencing (4).

The successful demonstration of DNA methylation analysis from archived blood spots will greatly facilitate future population-based epigenetic studies that may wish to examine retrospectively the association of specific epigenetic modifications at birth, with later health outcomes. Using such samples will provide a unique opportunity to investigate the role of epigenetics on the developmental origins of health and disease (DOHaD) (12). By demonstrating the ability to analyze DNA methylation from dried blood Guthrie spots, we have expanded their current use to include DNA methylation analyses. This also has implications on planning and establishing biospecimen collections for future cohort studies (13) that may look into the role of epigenetics, as dried blood spots are more amenable to storage at room temperature and transport between laboratories. Although bisulfite PCR and sequencing is considered the gold standard for DNA methylation analysis, this methodology will be applicable to other bisulfite-based assays, such as methylation-specific PCR (14), combined bisulfite restriction analysis (COBRA) (15), and others (reviewed in Reference 16). Not only will this allow researchers to link DNA methylation profile at birth to later disease, but also offers the opportunity for prospective collection of dried blood Guthrie spots for future DNA methylation analyses.

\section{ACKNOWLEDGMENTS}

N.C.W. is supported by the Phillip Desbrow Postdoctoral Fellowship from the Leukaemia Foundation of Australia. J.M.C. and R.S. are both R.D. Wright Research Fellows of the National Health and Medical Research Council (NHMRC) of Australia.

\section{COMPETING INTERESTS STATEMENT}

The authors declare no competing interests.

\section{REFERENCES}

1. Keffler, S., R. Denmeade, and A. Green. 1994. Neonatal screening for phenylketonuria: evaluation of an automated enzymatic method. Ann. Clin. Biochem. 31:134-139.

2. Audrezet, M.P., B. Costes, N. Ghanem, P. Fanen, C. Verlingue, J.F. Morin, B. Mercier, M. Goossens, and C. Ferec. 1993. Screening for cystic fibrosis in dried blood spots of newborns. Mol. Cell. Probes 7:497-502.

3. Clark, S.J., J. Harrison, C.L. Paul, and M. Frommer. 1994. High sensitivity mapping of methylated cytosines. Nucleic Acids Res. 22:2990-2997.

4. Millar, D.S., P.M. Warnecke, J.R. Melki, and S.J. Clark. 2002. Methylation sequencing from limiting DNA: embryonic, fixed, and microdissected cells. Methods 27:108-113.

5. Warnecke, P.M., C. Stirzaker, J. Song, C. Grunau, J.R. Melki, and S.J. Clark. 2002. Identification and resolution of artifacts in bisulfite sequencing. Methods 27:101-107.

6. Makowski, G.S., E.L. Davis, and S.M. Hopfer. 1996. The effect of storage on Guthrie cards: implications for deoxyribonucleic acid amplification. Ann. Clin. Lab. Sci. 26:458-469.

7. Ehrich, M., M.R. Nelson, P. Stanssens, M. Zabeau, T. Liloglou, G. Xinarianos, C.R.
Cantor, J.K. Field, and D. van den Boom. 2005. Quantitative high-throughput analysis of DNA methylation patterns by base-specific cleavage and mass spectrometry. Proc. Natl. Acad. Sci. USA 102:15785-15790.

8. Bock, C., S. Reither, T. Mikeska, M. Paulsen, J. Walter, and T. Lengauer. 2005. BiQ Analyzer: visualization and quality control for DNA methylation data from bisulfite sequencing. Bioinformatics 21:4067-4068.

9. Ehrich, M., S. Zoll, S. Sur, and D. van den Boom. 2007. A new method for accurate assessment of DNA quality after bisulfite treatment. Nucleic Acids Res. 35:e29.

10. Gokul, G., B. Gautami, S. Malathi, A.P. Sowjanya, U.R. Poli, M. Jain, G. Ramakrishna, and S. Khosla. 2007. DNA methylation profile at the DNMT3L promoter: a potential biomarker for cervical cancer. Epigenetics 2:80-85.

11. Heijmans, B.T., D. Kremer, E.W. Tobi, D.I. Boomsma, and P.E. Slagboom. 2007. Heritable rather than age-related environmental and stochastic factors dominate variation in DNA methylation of the human IGF2/H19 locus. Hum. Mol. Genet. 16:547-554.

12. Waterland, R.A. and K.B. Michels. 2007. Epigenetic epidemiology of the developmental origins hypothesis. Annu. Rev. Nutr. 27:363-388.

13. Brown, R.C., T. Dwyer, C. Kasten, D. Krotoski, Z. Li, M.S. Linet, J. Olsen, P. Scheidt, and D.M. Winn. 2007. Cohort profile: the International Childhood Cancer Cohort Consortium (I4C). Int. J. Epidemiol. 36:724-730.

14. Cottrell, S.E. and P.W. Laird. 2003. Sensitive detection of DNA methylation. Ann. N.Y. Acad. Sci. 983:120-130.

15. Xiong, Z. and P.W. Laird. 1997. COBRA: a sensitive and quantitative DNA methylation assay. Nucleic Acids Res. 25:2532-2534.

16. Fraga, M.F. and M. Esteller. 2002. DNA methylation: a profile of methods and applications. BioTechniques 33:632-649.

17.Coolen, M.W., A.L. Statham, M. GardinerGarden, and S.J. Clark. 2007. Genomic profiling of $\mathrm{CpG}$ methylation and allelic specificity using quantitative high-throughput mass spectrometry: critical evaluation and improvements. Nucleic Acids Res. 35:e119.

Received 12 December 2007; accepted 26 June 2008.

Address correspondence to Nicholas C. Wong, Developmental Epigenetics, Murdoch Childrens Research Institute, Royal Children's Hospital, Parkville, Vic 3052, Australia.e-mail:nick.wong@mcri.edu.au

To purchase reprints of this article, contact: Reprints@BioTechniques.com 